Artículo científico

Volumen 30(3):631-646. Septiembre-diciembre, 2019 e-ISSN 2215-3608, doi:10.15517/am.v30i3.36184 http://www.revistas.ucr.ac.cr/index.php/agromeso

\title{
Patogenicidad y autodiseminación de cepas promisorias de hongos entomopatógenos sobre Rhynchophorus palmarum L. (Coleoptera: Dryophthoridae)
}

\section{Pathogenicity and autodissemination of promising entomopathogenic fungi strains on Rhynchophorus palmarum L. (Coleoptera: Dryophthoridae)}

\author{
Guillermo Adolfo León-Martínez², Juan Carlos Campos-Pinzón², Jorge Humberto Arguelles-Cárdenas ${ }^{2}$
}

1 Recepción: 13 de febrero, 2019. Aceptación: 22 de abril, 2019. Este trabajo formó parte de un proyecto de investigación desarrollado en la Corporación Colombiana de Investigación Agropecuaria (AGROSAVIA), Colombia.

2 Corporación Colombiana de Investigación Agropecuaria (AGROSAVIA), Centro de Investigación La Libertad Km. 17 Vía Villavicencio - Puerto López. Villavicencio, Colombia. gleon@agrosavia.co (autor para correspondencia; https://orcid.org/0000-0001-6041-6305), jcampos@agrosavia.co,jarguelles@agrosavia.co

\section{Resumen}

Introducción. En Latinoamérica, el picudo (Rhynchophorus palmarum L.) es plaga de importancia en palma de aceite y cocotero, porque se asocia con la transmisión de anillo rojo (AR) y la pudrición del cogollo (PC), enfermedades que causan altos costos de manejo y mortalidad de plantaciones; los entomopatógenos Metarhizium anisopliae y Beauveria bassiana (Hypocreales: Clavicipitaceae) representan una alternativa de control microbiológico. Objetivo. El objetivo de este trabajo fue determinar la patogenicidad de cepas promisorias de M. anisopliae y B. bassiana sobre adultos de $R$. palmarum, y evaluar la diseminación de las mejores cepas desde adultos machos del picudo inoculados hacia hembras sin inocular. Materiales y métodos. El estudio se desarrolló en dos fases en el Centro de Investigación La Libertad de la Corporación Colombiana de Investigación Agropecuaria (AGROSAVIA), Departamento del Meta, Colombia, mediante arreglos factoriales en diseños completamente al azar; en la primera, se evaluaron cuatro cepas de $M$. anisopliae, una de $B$. bassiana y un producto comercial a base de $M$. anisopliae; en la segunda, para determinar la autodiseminación del entomopatógeno, se evaluaron las dos cepas de mayor eficacia del primer experimento (CPMa1104 y Mt008), mediante dos métodos de aplicación a machos (inmersión y polvo). Resultados. La cepa CPMa1104 mostró

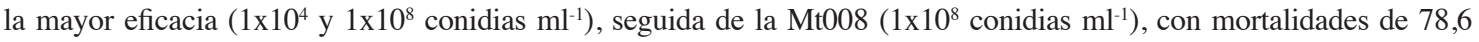
$\%$ y $67,9 \%$, respectivamente. Se observó capacidad de diseminación por contacto de M. anisopliae cepas CPMa1104 y Mt008, desde adultos machos inoculados hacia hembras sin inocular. La mortalidad de las hembras alcanzó 100 $\%$ cuando los machos fueron inoculados con la cepa CPMa1104 aplicada en polvo. Conclusión. Se comprobó la diseminación de las cepas CPMa1104 y Mt008 de M. anisopliae por machos inoculados hacia hembras sin inocular, lo cual convierte el uso de este entomopatógeno en una alternativa promisoria de control.

Palabras clave: control biológico, organismos patógenos, Elaeis guineensis.

\begin{abstract}
Introduction. In Latin America, the weevil (Rhynchophorus palmarum L.) is an important pest of oil palm and coconut palm because it is associated with the transmission of red ring (RRD) and bud rot (BR); diseases that cause high
\end{abstract}


management costs and mortality of plantations; the entomopathogens Metarhizium anisopliae and Beauveria bassiana (Hypocreales: Clavicipitaceae) represent an microbiological control alternative. Objective. The objective of this work was to determine the pathogenicity of promising strains of M. anisopliae and B. bassiana on adults of $R$. palmarum and to evaluate the spread of the best strains, from inoculated weevil male adults to not-inoculated females. Materials and methods. The study was carried out in two phases in the La Libertad Research Center of the Corporación Colombiana de Investigación Agropecuaria (AGROSAVIA), Department of Meta, Colombia through factorial arrangements in completely random designs; in the first one, four strains of M. anisopliae, one of B. bassiana, and one commercial product based on $M$. anisopliae were evaluated; in the second one, to determine the self-dissemination of the entomopathogen, the two highest effectiveness strains from the first experiment (CPMa1104 and Mt008) were evaluated by two methods of application to males (immersion and powder). Results. The CPMa1104 strain showed the highest efficacy $\left(1 \times 10^{4}\right.$ and $1 \times 10^{8}$ conidia $\left.\mathrm{ml}^{-1}\right)$, followed by Mt008 $\left(1 \times 10^{8}\right.$ conidia $\left.\mathrm{ml}^{-1}\right)$ with mortalities of $78.6 \%$ and $67.9 \%$, respectively. Contact dissemination capacity of $M$. anisopliae strains CPMa1104 and Mt008 was observed from inoculated male adults to non-inoculate females. Female mortality reached $100 \%$ when the males were inoculated with powdered CPMa1104. Conclusion. The dissemination of CPMa1104 and Mt008 strains of M. anisopliae by inoculated males to non-inoculated females was tested, which makes the use of this entomopathogen a promising control alternative.

Keywords: biological control, pathogenic organisms, Elaeis guineensis.

\section{Introducción}

La producción de aceite de palma en Latinoamérica durante el período 2001 a 2014, tuvo un crecimiento del 143,5 \% . Los países con mayor área sembrada fueron Colombia, Ecuador, Honduras, Guatemala y Brasil, los cuales aportaron cerca del $84 \%$ de la producción total de la región, con un rendimiento promedio de $3,6 \mathrm{t} \mathrm{ha-1}$, muy por debajo de promedios de referencia para este cultivo entre 20 y 25 toneladas por hectárea, debido principalmente a problemas fitosanitarios presentes en la región (González-Cárdenas, 2016). Los países latinoamericanos con mayor exportación de aceite de palma fueron Guatemala, Honduras, Colombia y Ecuador, los cuales contribuyeron con el 74,7 \% del total; para el año 2017, Latinoamérica exportaba el $6 \%$ de la producción mundial (4,1 millones de toneladas) de aceite de palma, destacando Colombia con 1,6 millones de t, Ecuador con $543000 \mathrm{t}$, Guatemala con 740000 t, Honduras con 545000 t, Brasil con 410000 t y Costa Rica con 270000 t.

En Centroamérica el área sembrada en palma aceitera sobrepasa las 370000 ha, cifra que va en aumento por la expansión de la demanda global de aceite de palma y a que, recientemente, países como Costa Rica, Guatemala y Honduras han intensificado su producción para la obtención biocombustibles; según la Federación Nacional de Palmeros de Honduras, siete de cada diez plantas producen biodiesel en dicho país. Para el año 2014 en Guatemala se reportaron 130000 ha de palma, en Honduras algo más de 165000 ha y en Costa Rica se superan las 66000 ha sembradas (Picado, 2017).

Colombia es el cuarto productor de aceite de palma del mundo y el primer productor en América (Fedepalma, 2016). En el país, la palma de aceite (E. guineensis Jacq.) se encuentra distribuida en cuatro zonas productivas; norte, centro, oriente y sur occidente, con características agroecológicas diferentes (Dangon, 2014). En el año 2016, se reportó un área sembrada de 483733 ha, con una producción de 1272521 t (FEDEPALMA, 2016).

Los principales productores de coco son los países asiáticos, aunque sus rendimientos han bajado un $60 \%$, debido principalmente al envejecimiento de las plantaciones; y mientras la demanda global de este producto básico para alimentación, jabones, combustibles y cosméticos aumenta hasta en un $10 \%$, la productividad del sector 
solamente crece al $2 \%$ (FAO, 2013), lo cual es un indicativo de gran potencial para el sector productivo de coco en Latinoamérica.

Colombia ocupa el tercer lugar en producción de coco en Suramérica con 130662 t, después de Venezuela y Brasil; la productividad de coco alcanzada por Colombia $\left(7,38 \mathrm{t} \mathrm{ha}^{-1}\right)$ supera los promedios mundiales registrados de 5 t ha $^{-1}$ (FAOSTAT, 2015). La principal zona de producción se concentra en las regiones Caribe y Pacífica. En la región Pacífica, los departamentos de Cauca, Chocó, Nariño y Valle del Cauca registraron una producción de 82 511 t para el año 2014; el departamento de Nariño ocupó el primer puesto con el 40,18 \% de la producción nacional. En la región Caribe, el departamento de Córdoba ocupó el primer lugar con el 22,79 \% de la producción nacional y una producción total de 29539 t para el 2014 (Agronet, 2014).

El picudo Rhynchophorus palmarum L. (Coleoptera: Dryophthoridae), también conocido como picudo negro de las palmas, picudo americano de las palmas (PAP) y denominado regionalmente gualpa, es un insecto-plaga de importancia económica para los cultivos de palma de aceite y cocotero en América Latina y el Caribe (Hagley, 1963). Es el principal agente transmisor del nematodo Bursaphelenchus cocophilus Cobb. (antes Rhadinaphelenchus cocophilus), agente causal de la enfermedad anillo rojo-hoja corta (AR) de las palmáceas; estos dos organismos conforman el complejo anillo rojo-picudo americano de las palmas (AR-PAP), causante de la muerte de cientos de miles de palmas en toda América Latina (Chinchilla, 1988; Genty, 1988; Griffith, 1987; Mora, et al., 1994; Mora, 2007; Grueso y Betancourth, 2009; Aldana et al., 2011).

El complejo AR-PAP es una de las principales limitantes fitosanitarias de los cultivos de palma aceitera y cocotero. El anillo rojo causa grandes pérdidas económicas, es una enfermedad endémica de Latinoamérica y, en Colombia, se presenta con mayor severidad en la costa Pacífica; las pérdidas ocasionadas en plantaciones de palma de aceite en los Llanos Orientales fueron de aproximadamente ocho millones de dólares entre 1990 y 2004, y se estima que, en los departamentos del Cauca y Nariño, ocasiona la muerte del $5 \%$ de las palmas por año (Aldana et al., 2010). Adicionalmente, $R$. palmarum se asocia con la transmisión mecánica de la pudrición del cogollo en palma (PC), debido a que se alimenta de plantas afectadas y también puede atacarlas directamente, originando daño en sus tejidos, ya que las larvas se alimentan del tejido blanco del cogollo, del meristemo y de las bases peciolares, lo cual causa la muerte a las palmas afectadas (Calvache et al., 1994; Aldana et al., 2007). El complejo fitosanitario PC/PAP, ha causado en el país pérdidas económicas estimadas en USD \$5,5 billones, de los cuales se considera que USD \$2 billones corresponden a las pérdidas producidas por la afectación de unas 70000 ha en Tumaco (Nariño), Puerto Wilches (Santander) y Cantagallo, (Bolívar) (MADR et al., 2014). El complejo AR-PAP en el cultivo del cocotero fue detectado en la Costa Atlántica en el departamento de Sucre en 1962 y, posteriormente, en 1967 registró daños severos por $R$. palmarum en los Departamentos de Cauca y Nariño, con pérdidas del $50 \%$ de las plantaciones. A finales de los años ochenta se estimaron daños del $90 \%$ de los cultivos en Tumaco, región de gran importancia para el cultivo del cocotero, y desde dicha época el complejo se encuentra establecido en la región (Grueso y Betancourth, 2009).

El control de R. palmarum, está basado en el uso de insecticidas químicos altamente tóxicos, lo que trae como consecuencia problemas en la salud humana, contaminación del ambiente y resistencia del insecto a estos productos; el uso de trampas con feromona de agregación para la captura de adultos de R. palmarum (Oehlschlager, 2007) y la erradicación de palmas afectadas por la enfermedad (AR y PC), acompañado con la eliminación manual de los estados inmaduros, es la alternativa más utilizada para el manejo de estos complejos fitosanitarios (Aldana et al., 2011).

Las especies de hongos entomopatógenos Metarhizium anisopliae y Beauveria bassiana (Hypocreales: Clavicipitaceae) son las más estudiadas y utilizadas en el control biológico de insectos, su uso potencial como bioinsecticidas es reconocido sobre diferentes familias de coleóptera (Bustillo et al., 1999; Godonou et al., 2000; Kreutz et al., 2004; Nájera-Rincón et al., 2005; Gindin et al., 2006; Tinzaara, 2007; Pedrini et al., 2010). 
El hongo M. anisopliae es utilizado también para el control de otras plagas del cocotero como los escarabajos rinoceronte Oryctes rhinoceros L. (Coleoptera: Scarabaidae) (Gopal et al., 2002), Scapanes australis Biosd. (Coleoptera: Scarabaidae) (Prior et al., 1985) y el picudo negro de las palmas, Rhynchophorus bilineatus Montrouzier (Coleoptera: Dryophthoridae) (Prior et al., 1985). Se conoce que el hongo B. bassiana es un agente entomopatógeno del picudo del cocotero, Pantorhytes plutus Oberthür (Coleoptera: Dryophthoridae) (Prior et al., 1988); su alta capacidad insecticida ha sido reportada en el control de Rhynchophorus ferrugineus (Faleiro, 2006; Gindin et al., 2006) y el picudo negro de las palmas R. palmarum (Costa et al., 2011; Alvarado et al., 2013). Cepas de $M$. anisopliae con alta capacidad insecticida han sido reportadas controlando insectos como Cosmopolites sordidus (Castrillón, 2000), además de otras plagas del orden Coleoptera en palma africana, por lo que, constituye un potencial para desarrollarlos como una alternativa de manejo biológico para $R$. palmarum y otras plagas en palma aceitera (Alvarado et al., 2013).

Aspersiones mensuales de $M$. anisopliae a una concentración de $1 \times 10^{8}$ conidios $\mathrm{ml}^{-1}$, pueden reducir el número de adultos de $R$. palmarum en un 48,2 \% en épocas de lluvia (julio), y en un $60 \%$, con cinco aplicaciones mensuales, entre enero y junio; mientras que en el testigo únicamente con trampeo tradicional se disminuyó en un $20 \%$ (Mora, 2007).

El hongo $M$. anisopliae causa mortalidades significativas de adultos de $R$. palmarum, por el método de inmersión a una concentración de $10^{7}$ conidios $\mathrm{ml}^{-1}$ en condiciones de laboratorio, con una tasa de infección del 85,7 $\%$ y esporulación hasta del 60,7 \%, luego de veinticuatro días de aplicado el tratamiento (Alvarado et al., 2013).

La Corporación Centro de Investigación en Palma de Aceite (Cenipalma) ha demostrado la virulencia y patogenicidad de M. anisopliae sobre adultos de R. palmarum, hasta en 87 \% (Aldana et al., 2011). La evaluación de diferentes cepas de $M$. anisopliae, presentó una alta patogenicidad y virulencia sobre adultos de $R$. palmarum, con

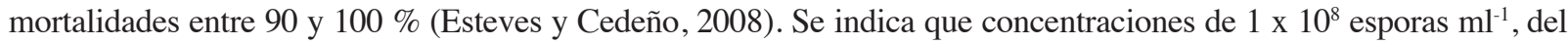
hongo M. anisopliae, causan mortalidades del $100 \%$ después de treinta días sobre adultos de R.ferrugineus (Gindin et al., 2006).

La auto diseminación de entomopatógenos entre insectos adultos de la misma especie, ya ha sido estudiada con resultados favorables. Cuando se investigó sobre la transmisión horizontal del B. bassiana entre adultos tratados y no tratados del escarabajo de la corteza de abeto Ips typographus (Coleoptera: Scolytidae), se encontró que la transmisión de B. bassiana desde individuos contaminados hacia no contaminados fue del $99 \%$, después de diecinueve días; un contacto simple entre un macho tratado con 2,0x10 conidias por individuo y una hembra no tratada fue suficiente para transmitir una dosis letal de B. bassiana (Kreutz et al., 2004); además se demostró la auto diseminación de $B$. bassiana entre los cucarrones, y se encontraron reducciones significativas en la longitud de las galerías construidas por las hembras y el número de larvas y pupas de la descendencia; cuando se utilizó feromona tratada con $B$. bassiana, se observaron reducciones significativas en las poblaciones del cucarrón, en el número de perforaciones y en la longitud de las galerías maternas en los troncos de abeto (Kreutz et al., 2004).

Se encontró una transmisión horizontal de campo de B. bassiana entre los picudos del plátano Cosmopolites sordidus (Coleoptera: Dryophthoridae) con el uso de feromonas de agregación, al comprobar que picudos infectados lograban transmitir el patógeno hacia individuos sanos, con una eficacia del $52 \%$; hubo mayor mortalidad en las parcelas donde se aplicó $B$. bassiana en combinación con feromona en comparación con los tratamientos donde se aplicó solo el entomopatógeno (Tinzaara et al., 2007), por lo cual, la feromona de agregación es una potencial herramienta para mejorar la diseminación de B. bassiana para el control de C. sordidus.

El objetivo del presente trabajo fue determinar la patogenicidad de diferentes cepas de los hongos entomopatógenos $M$. anisopliae y B. bassiana sobre adultos de $R$. palmarum y evaluar la diseminación de las mejores cepas desde adultos machos del picudo inoculados hacia hembras sin inocular. 


\section{Materiales y métodos}

Para establecer el efecto de los entomopátógenos Metarhizium anisopliae y Beauveria bassiana, sobre adultos de $R$. palmarum, se realizaron pruebas de patogenicidad en el laboratorio de entomología del Centro de Investigación La Libertad de AGROSAVIA (Corporación Colombiana de Investigación Agropecuaria), ubicado en el municipio de Villavicencio (Meta), Colombia. Para el desarrollo de las pruebas en laboratorio, se utilizó la metodología de inmersión de adultos en esporas propuesta por González et al. (1993). La selección se realizó sobre cepas de M. anisopliae, probadas por su alta capacidad virulenta sobre adultos de R. palmarum, y con registro de accesión en los bancos de especies entomopatógenas de AGROSAVIA y CENIPALMA (Centro de Investigación en Palma de Aceite); adicionalmente, se utilizó una cepa autóctona de B. bassiana colectada en los llanos orientales de Colombia, así como una formulación comercial registrada para el control de R. palmarum.

\section{Obtención, reactivación y multiplicación de cepas de $M$. anisopliae y $B$. bassiana}

Para M. anisopliae se seleccionaron cuatro cepas: dos de ellas, las accesiones CPMa1104 y CPMa1105 (Figura 1A), provenientes de la Colección de Microorganismos Asociados a la Palma de Aceite de Cenipalma, registradas con el número 182 en el Registro Único Nacional de Colecciones Biológicas (RNC); las dos restantes, accesiones Mt040 y Mt008 (Figura 1B), procedentes del Banco de Germoplasma de Microorganismos con Interés en Control Biológico de la Nación, administrado por AGROSAVIA en el Centro de Investigación Tibaitatá.
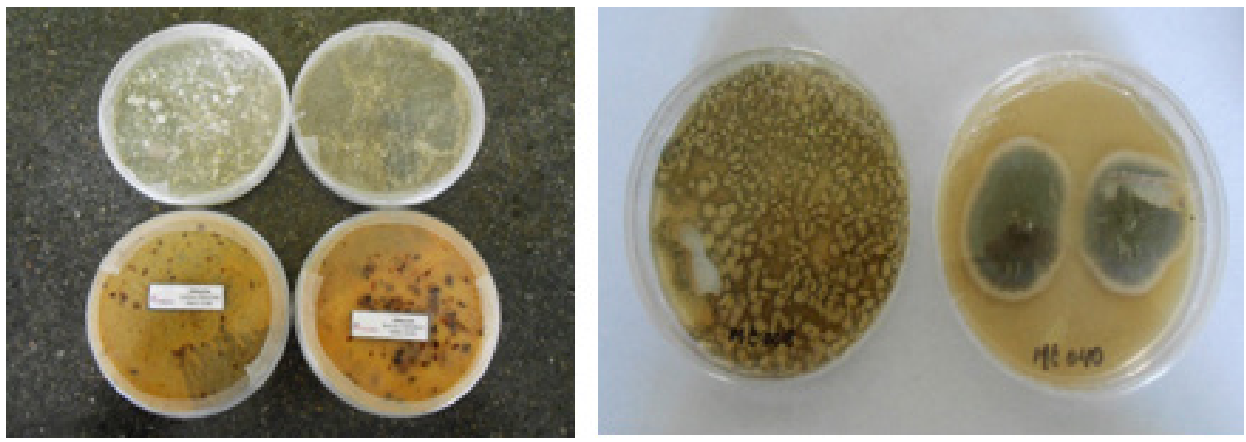

Figura 1. A. Accesiones CPMa1104 y CPMa1105 de M. anisopliae provenientes de la colección de microorganismos de Cenipalma, Laboratorio de Microorganismos Entomopatógenos, Bogotá, Colombia, 2016. B. Accesiones Mt008 y Mt040 de M. anisopliae, provenientes del banco de germoplasma de microorganismos con interés biológico de AGROSAVIA, Centro de Investigación Tibaitatá, Colombia. 2016.

Figure 1. A. CPMa1104 and CPMa1105 M. anisopliae accessions from the microorganism collection of Cenipalma, Laboratory of Entomopathogenic Microorganisms, Bogotá, Colombia, 2016. B. Mt008 and Mt040 accessions of M. anisopliae, from the germplasm microorganism of biological interest bank of AGROSAVIA, Tibaitatá Research Center, Colombia. 2016.

La cepa de B. bassiana utilizada en este trabajo (Figura 2), fue recuperada y aislada de un adulto de $R$. palmarum, recolectado en la plantación comercial de palma de aceite del Centro de Investigación La Libertad. El aislamiento de esta cepa se realizó en medio de cultivo PDA. Luego de este proceso fue ingresada al banco de germoplasma de microorganismos con interés en control biológico de la nación, y se registró con el código de accesión Bv063. 


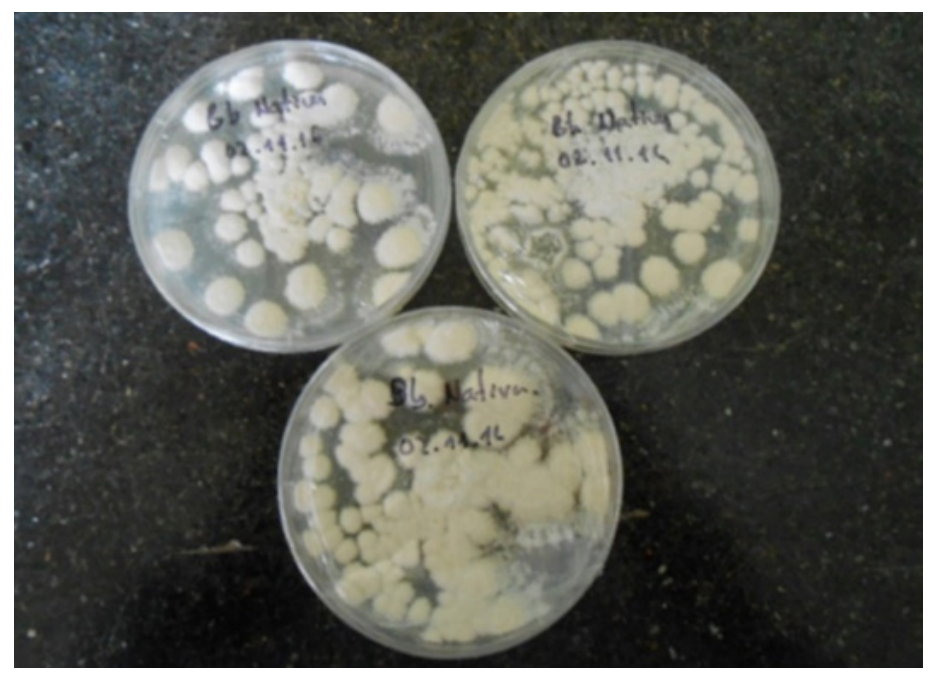

Figura 2. Cepa nativa de B. bassiana accesión Bv063, recuperada de Rhynchophorus palmarum L. y aislada en medio PDA. Laboratorio de Entomología. Centro de Investigación La Libertad, Villavicencio, Meta, Colombia. 2017.

Figure 2. Native strain of B. bassiana Bv063 accession recovered from Rhynchophorus palmarum L. and isolated on PDA medium. Entomology Laboratory of La Libertad Research Center, Villavicencio, Meta, Colombia. 2017.

La reactivación de las cepas se realizó sobre adultos de $R$. palmarum a una concentración ajustada de $3 \times 10^{8}$ conidios $\mathrm{ml}^{-1}$, bajo condiciones de laboratorio, a temperatura promedio de $25^{\circ} \mathrm{C} \pm 2{ }^{\circ} \mathrm{C}$ y humedad relativa de 70 $\% \pm 5 \%$. Los individuos muertos se individualizaron dentro de cámaras húmedas consistentes en cajas petri de 90 $\mathrm{mm}$, con papel filtro humedecido con $0,2 \mathrm{ml}$ de agua destilada estéril y una lámina porta objetos esterilizada, sobre la cual se colocó el insecto muerto; de los insectos esporulados se realizó el aislamiento de cada una de las cepas en medio de cultivo Yeast Mold Agar (YM), para su posterior multiplicación.

La multiplicación se realizó en medio sólido (50 g de arroz y $80 \mathrm{ml}$ de agua) dentro de botellas de vidrio de $375 \mathrm{ml}$; el sustrato se esterilizó y luego se inoculó con $1 \mathrm{ml}$ de suspensión de conidios de cada cepa. Posteriormente, se mantuvo a una temperatura de $27{ }^{\circ} \mathrm{C}$ y humedad relativa de $75 \%$, durante 30 días hasta observar crecimiento micelial y esporulación.

Luego de la purificación y reactivación de las cepas seleccionadas, el trabajo comprendió dos fases. En la primera fase se evaluó la mortalidad causada por cepas promisorias de M. anisopliae y $B$. bassiana en condiciones de laboratorio, para establecer su patogenicidad a diferentes concentraciones sobre adultos del picudo $R$. palmarum. En la segunda fase, se determinó la autodiseminación de dos cepas de $M$. anisopliae causada por adultos machos de R. palmarum inoculados con esporas del hongo, hacia hembras $\sin$ inocular.

\section{Fase 1. Determinación del efecto de los hongos entomopatógenos sobre $R$. palmarum}

Para determinar el efecto de los hongos entomopatógenos, se colectaron en campo adultos de R. palmarum y se colocaron en cuarentena durante cinco días, para apartar insectos enfermos antes de aplicar los tratamientos. Las esporas de las cepas de M. anisopliae y B. bassiana reactivadas y multiplicadas en laboratorio, se separaron en medio húmedo mediante lavado con Tween ${ }^{\circledR} 80$ al 0,1 \% y agitación con Vortex. La suspensión obtenida de cada cepa se cuantificó mediante recuento en cámara de Neubauer y, a partir de estas, se ajustaron las concentraciones para cada uno de los tratamientos a evaluar. 
Se evaluaron cuatro cepas de M. anisopliae (CPMa1104, CPMa1105, Mt040 y Mt008), una cepa de B. bassiana nativa (Bv063) y un producto comercial (Anisafer WP), el cual es bioplaguicida con Registro ICA y composición garantizada de $M$. anisopliae $1 \times 10^{8}$ esporas $\mathrm{g}^{-1}$ y Bacillus popiliae $1 \times 10^{4} \mathrm{UFC}^{-1}$. En el Cuadro 1 se describen los tratamientos y concentraciones evaluadas.

Cuadro 1. Cepas entomopatógenas y concentraciones evaluadas para determinar la patogenicidad sobre individuos adultos de $R$. palmarum. Laboratorio de Entomología, Centro de Investigación La Libertad, Villavicencio, Meta, Colombia. 2017.

Table 1. Entomopathogenic strains and concentrations evaluated to determine the pathogenicity on $R$. palmarum adult individuals. Entomology Laboratory, La Libertad Research Center, Villavicencio, Meta, Colombia. 2017.

\begin{tabular}{|c|c|c|c|c|}
\hline Entomopatógeno & Código o nombre & Concentración & Repeticiones & $\begin{array}{c}\text { Individuos por } \\
\text { Tratamiento }\end{array}$ \\
\hline Metarhizium anisopliae & $\begin{array}{c}\text { CPMa1104 } \\
\text { CPMa1105 } \\
\text { Mt040 } \\
\text { Mt008 }\end{array}$ & \multirow[t]{2}{*}{$\begin{array}{c}1 \times 10^{4} \\
1 \times 10^{6} \\
1 \times 10^{8} \\
\text { conidias } \mathrm{ml}^{-1}\end{array}$} & \multirow{4}{*}{ Tres por tratamiento } & \multirow{4}{*}{ Treinta } \\
\hline Beauveria bassiana & Bv063 & & & \\
\hline Producto comercial a base $M$. anisopliae & Anisafer WP & $1 \times 10^{8}$ esporas $\mathrm{g}^{-1}$ & & \\
\hline Control & Sin aplicación & & & \\
\hline
\end{tabular}

Para este experimento, se utilizó un arreglo factorial aumentado $(5 \times 3+2)$ : cinco cepas a tres dosis cada una, más dos testigos (testigo comercial y control), para un total de diez y siete tratamientos, en un diseño experimental completamente al azar con tres repeticiones por tratamiento, para un total de 51 unidades experimentales; cada una de ellas estuvo constituida de treinta individuos.

Para la inoculación, se utilizó el método de inmersión por cinco minutos en $50 \mathrm{ml}$ de cada una de las concentraciones. Los adultos de $R$. palmarum inoculados se confinaron en cajas transparentes de 48 onzas y se alimentaron con caña de azúcar, la cual se cambió semanalmente durante el tiempo de evaluación. El experimento se mantuvo en condiciones de laboratorio a una temperatura promedio de $27 \pm 5^{\circ} \mathrm{C}$ y humedad relativa de $75 \pm 5 \%$ durante 21 días. Los insectos muertos se individualizaron, de acuerdo con cada tratamiento, en cámaras húmedas, cajas petri ya descritas; para cada tratamiento se calculó la mortalidad expresada en porcentaje. Para establecer el efecto de las cepas, de sus concentraciones y de su interacción sobre la mortalidad de individuos de R. palmarum, se realizó un análisis de varianza, mediante el procedimiento GLIMMIX (modelos lineales generalizados mixtos) del paquete SAS® (SAS Institute Inc., 2016) versión 9.4, que permite analizar los datos con falta de homocedasticidad y normalidad, debido a que en este caso, no se cumplieron los anteriores supuestos que requieren los modelos lineales clásicos del análisis de varianza.

\section{Fase 2. Autodiseminación de M. anisopliae desde machos hacia hembras de $R$. palmarum}

Para determinar la capacidad de autodiseminación por contacto de $M$. anisopliae, desde adultos machos de $R$. palmarum inoculados con esporas del hongo hacia hembras sin inocular, se probaron las cepas de mayor eficacia resultantes de la fase 1 (CPMa1104 y Mt008). Para ello, se capturaron adultos de R. palmarum en campo y se colocaron en cuarentena dentro de cajas plásticas durante cinco días; posteriormente, las hembras se confinaron en contenedores plásticos con trozos de caña de azúcar como alimentación. 
Los tratamientos a evaluar consistieron someter a los machos de R. palmarum a: inmersión en suspensiones de esporas por $5 \mathrm{~min}$, e inoculación en polvo durante $5 \mathrm{~min}$; para la inmersión de machos se usaron suspensiones de $50 \mathrm{ml}$ con esporas de las cepas CPMa1104 y Mt008 a concentraciones de $1 \times 10^{8}$ conidias $\mathrm{ml}^{-1}$, y la inoculación en polvo se realizó con $5 \mathrm{~g}$ de esporas de cada cepa por contacto directo con los machos.

Una vez inoculados los machos, se trasladaron a los contenedores con las hembras para permitir su contacto. Se dejaron en confinamiento durante cinco días y luego se retiraron los machos para evaluar la mortalidad de las hembras en cada tratamiento. La mortalidad en las hembras de R. palmarum, se registró durante los veintiún días posteriores al confinamiento con los machos, para determinar el efecto de cada tratamiento; los insectos muertos se individualizaron en cámaras húmedas (cajas de Petri previamente descritas), para favorecer la esporulación del hongo y así observar el desarrollo de la enfermedad.

El experimento correspondió a un arreglo factorial 2 x 2 x 3, dos cepas (CPMa1104 y Mt008), dos métodos de aplicación (inmersión y polvo) y tres relaciones macho/hembra (1:1, 1:5 y 1:10), para un total de doce tratamientos en un diseño completamente al azar con tres repeticiones por tratamiento, con 36 unidades experimentales y diez hembras por unidad experimental.

Para determinar la capacidad de autodiseminación del entomopatógeno desde machos inoculados hacia hembras sin inocular, al igual que en el anterior experimento, se realizó un análisis de varianza, mediante el procedimiento GLIMMIX (modelos lineales generalizados mixtos) del paquete SAS ${ }^{\circledR}$ (SAS Institute Inc., 2016) versión 9.4, para establecer el efecto de las cepas, del método de aplicación, de la relación de sexos y su interacción sobre la mortalidad.

\section{Resultados}

\section{Fase 1. Determinación del efecto de los hongos entomopatógenos sobre $R$. palmarum}

Para los tratamientos evaluados, se registraron mortalidades de adultos de $R$. palmarum entre el 10 y el $90 \%$. La esporulación en los insectos muertos por efecto de los entomopatógenos se observó en todos los tratamientos de $M$. anisopliae (cepas CPMa1104, CPMa1105, MT040 y MT008), pero no ocurrió en los tratamientos de $B$. bassiana cepa Bv063, ni con el producto comercial a base de $M$. anisopliae. La esporulación sucedió a través de los espiráculos, los espacios intersegmentales de la cutícula del cuerpo, las patas, las bases y terminales antenales y del rostrum de los adultos de R. palmarum (Figura 3).

De acuerdo con el análisis de varianza para los efectos de las cepas, las concentraciones evaluadas y la interacción cepa por concentración, la mortalidad de los individuos de $R$. palmarum presentó diferencias estadísticas significativas $(\alpha<0,01)$ entre las cepas $M$. anisopliae y $B$. bassiana, entre las dosificaciones evaluadas para estas cepas y entre la interacción cepa*concentración.

Al comparar las medias estimadas por mínimos cuadrados para la mortalidad (\%) provocada por las cepas y sus respectivas concentraciones en conidias $\mathrm{ml}^{-1}$, sobre los individuos de $R$. palmarum, se observó que los mayores porcentajes, se obtuvieron con la cepa CPMa1104 (M. anisopliae), mientras que la cepa CPMa1105 presentó la más baja mortalidad y las cepas Mt008, Mt040 (M. anisopliae) y Bv063 (B. bassiana), mostraron valores medios; el producto comercial generó una mortalidad baja (Cuadro 2).

Debido al alto número de combinaciones de cepas y sus concentraciones, y para facilitar la interpretación de los resultados, se decidió realizar un análisis de conglomerados, mediante el procedimiento CLUSTER (algoritmo de Ward) del software SAS®, para clasificar los tratamientos con base en las medias ajustadas por mínimos cuadrados. Este análisis permitió conformar cuatro grupos de tratamientos que se describieron mediante estadísticas básicas (media, valores mínimos y máximos y coeficiente de variación) (Cuadro 3). Como se pudo observar, la mayor mortalidad correspondió al grupo 4, que incluyó a los tratamientos de las cepas CPMa1104 (M. 


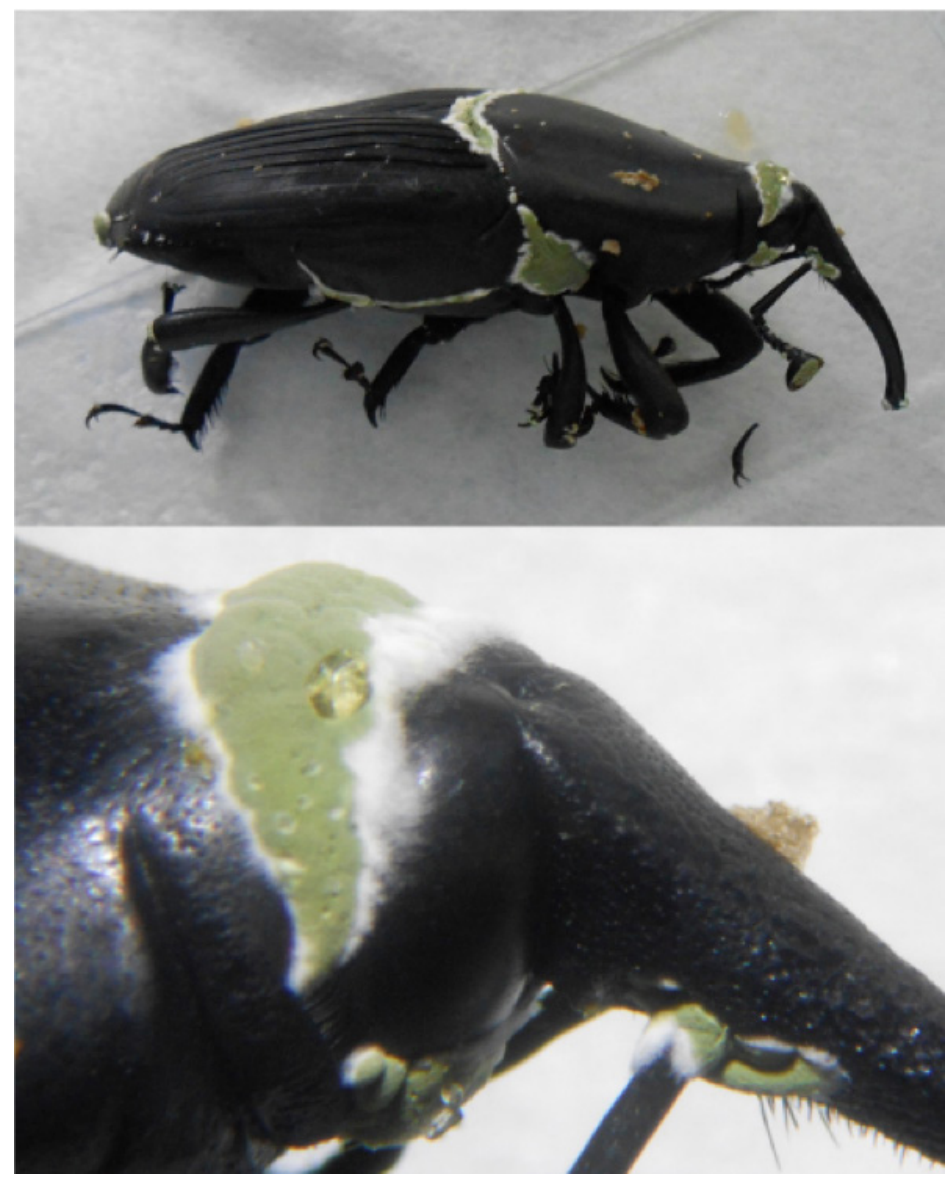

Figura 3. Esporulación del entomopatógeno M. anisopliae inoculado en adultos de RhynchophoruspalmarumL. Centro de Investigación La Libertad, Villavicencio, Meta, Colombia. 2017.

Figure 3. Sporulation of the M. anisopliae entomopathogen inoculated in adults of R. palmarum. La Libertad Research Center, Villavicencio, Meta, Colombia. 2017.

anisopliae) en concentraciones de $1 \times 10^{8}$ y $1 \times 10^{4}$ conidias $\mathrm{ml}^{-1}$ y $\mathrm{Mt} 008$ (M. anisopliae) en concentración $1 \times 10^{8}$. La mortalidad más baja correspondió al grupo 1 con los tratamientos que correspondieron al producto comercial, la cepa CPMa1105 (M. anisopliae) en concentraciones de $1 \times 10^{6}$ y $1 \times 10^{4}$ conidias $\mathrm{ml}^{-1}$, y al control sin aplicación. Los demás tratamientos incluidos en los grupos 2 y 3, presentaron mortalidades intermedias entre 23,3 y $60 \%$. De acuerdo con estos resultados, las cepas CPMa1104 y Mt008 (M. anisopliae) fueron seleccionadas para la fase 2 del estudio.

\section{Fase 2. Autodiseminación de $M$. anisopliae desde machos hacia hembras de $R$. palmarum}

Los resultados demostraron que los machos de $R$. palmarum inoculados con esporas de $M$. anisopliae de las cepas CPMa1104 y Mt008, lograron diseminar el entomopatógeno y contaminar por contacto a hembras no inoculadas, en condiciones de confinamiento y en las diferentes relaciones numéricas macho/hembra evaluadas. 
Cuadro 2. Mortalidad de adultos de Rhynchophorus palmarum L., por efecto de la inoculación de cepas de Metarhizium anisopliae o Beauveria bassiana. Centro de Investigación La Libertad, Villavicencio, Meta, Colombia. 2017.

Table 2. Adult mortality of Rhynchophorus palmarum, due to the inoculation of Metarhizium anisopliae or Beauveria bassiana strains. La Libertad Research Center, Villavicencio, Meta, Colombia. 2017.

\begin{tabular}{cccc}
\hline Tratamiento & Cepa & Concentración $\left(\right.$ conidias $\left.\mathbf{~ m l}^{\mathbf{1}}\right)$ & Mortalidad* $^{*}$ \%) \\
\hline 5 & CPMa1104 & $1 \times 10^{4}$ & 90,00 \\
4 & CPMa1104 & $1 \times 10^{8}$ & 80,00 \\
10 & Mt008 & $1 \times 10^{8}$ & 73,33 \\
6 & CPMa1104 & $1 \times 10^{6}$ & 60,00 \\
13 & Mt040 & $1 \times 10^{8}$ & 56,67 \\
15 & Mt040 & $1 \times 10^{6}$ & 56,67 \\
12 & Mt008 & $1 \times 10^{6}$ & 5323 \\
7 & CPMa1105 & $1 \times 10^{8}$ & 50,00 \\
3 & Bv063 & $1 \times 10^{6}$ & 43,33 \\
1 & Bv063 & $1 \times 10^{8}$ & 36,67 \\
2 & Bv063 & $1 \times 10^{4}$ & 36,67 \\
14 & Mt040 & $1 \times 10^{4}$ & 26,67 \\
8 & Mt008 & $1 \times 10^{4}$ & 23,33 \\
11 & P. comercial** & $1 \times 10^{8}$ & 13,33 \\
17 & CPMa 1105 & $1 \times 10^{4}$ & 13,33 \\
\hline
\end{tabular}

*Medias ajustadas por mínimos cuadrados, **Producto comercial a base de $M$. anisopliae / *Least-square adjusted averages, ** Commercial product based on M. anispliae.

Cuadro 3. Estadísticas descriptivas para la mortalidad de Rhynchophorus palmarum L. generada por cada grupo de tratamientos (cepas * concentración de conidios). Villavicencio, Meta, Colombia. 2017.

Table 3. Descriptive statistics for the mortality Rhynchophorus palmarum L. generated by each group of treatments (Strains *conidium concentration). Villavicencio, Meta, Colombia, 2017.

\begin{tabular}{|c|c|c|c|c|c|c|}
\hline Grupos & Cepas & Concentración & Media & Mínimo & Máximo & C.V. $(\%)$ \\
\hline \multirow{4}{*}{1} & P. comercial $* *$ & $1 \times 10^{8}$ & \multirow{4}{*}{9,8} & \multirow{4}{*}{2,53} & \multirow{4}{*}{13,33} & \multirow{4}{*}{51,96} \\
\hline & СРMa 1105 & $1 \times 10^{6}$ & & & & \\
\hline & СРМа 1105 & $1 \times 10^{4}$ & & & & \\
\hline & CONTROL & CERO & & & & \\
\hline \multirow{5}{*}{2} & Bv063 & $1 \times 10^{8}$ & \multirow{5}{*}{33,33} & \multirow{5}{*}{23,33} & \multirow{5}{*}{43,33} & \multirow{5}{*}{24,49} \\
\hline & $\mathrm{Bv063}$ & $1 \times 10^{4}$ & & & & \\
\hline & $\mathrm{Bv063}$ & $1 \times 10^{6}$ & & & & \\
\hline & $\mathrm{Mt} 008$ & $1 \times 10^{4}$ & & & & \\
\hline & $\mathrm{Mt} 040$ & $1 \times 10^{4}$ & & & & \\
\hline \multirow{5}{*}{3} & CPMa1104 & $1 \times 10^{6}$ & \multirow{5}{*}{55,33} & \multirow{5}{*}{50} & \multirow{5}{*}{60} & \multirow{5}{*}{6,87} \\
\hline & CPMa1105 & $1 \times 10^{8}$ & & & & \\
\hline & $\mathrm{Mt} 008$ & $1 \times 10^{6}$ & & & & \\
\hline & $\mathrm{Mt} 040$ & $1 \times 10^{8}$ & & & & \\
\hline & $\mathrm{Mt} 040$ & $1 \times 10^{6}$ & & & & \\
\hline \multirow{3}{*}{4} & CPMa1104 & $1 \times 10^{8}$ & \multirow{3}{*}{81,11} & \multirow{3}{*}{73,33} & \multirow{3}{*}{90} & \multirow{3}{*}{10,34} \\
\hline & CPMa1104 & $1 \times 10^{4}$ & & & & \\
\hline & Mt008 & $1 \times 10^{8}$ & & & & \\
\hline
\end{tabular}

***Producto comercial a base de M. anisopliae. C.V.: coeficiente de variación / ** Commercial product based on M. anispliae. C.V.: variation coefficient. 
De acuerdo con el análisis de varianza, se detectaron diferencias estadísticas significativas entre las categorías de los factores: cepa de M. anisopliae y método de aplicación (polvo e inmersión); la interacción cepa*método de aplicación también fue estadísticamente significativa. Al comparar el método de inoculación de $M$. anisopliae en polvo vs en inmersión, se presentó mayor eficacia de los tratamientos de inoculación en polvo sobre la mortalidad de las hembras, con valores entre 63,3 y $100 \%$, mientras que los tratamientos en inmersión acuosa de esporas presentaron mortalidades de hembras entre 16,67 y $30 \%$. Con respecto a la interacción entre cepa y método de aplicación, se encontró que CPMa1104 aplicada en polvo a los machos, fue diseminada y controló el $100 \%$ de las hembras de $R$. palmarum que fueron confinadas con los machos; este valor fue estadísticamente diferente a la cepa Mt008 aplicada a los machos en polvo, que alcanzó una mortalidad promedio de 72,2\% de hembras de $R$. palmarum, y también fue diferente estadísticamente a la media de los demás tratamientos, correspondientes a las cepas aplicadas a los machos mediante el método de inmersión, cuyo valor fue 23,33\%. En el Cuadro 4, se relacionan las medias ajustadas por mínimos cuadrados, de todos los tratamientos evaluados; como en el experimento anterior, se recurrió a un análisis de conglomerados, mediante el procedimiento CLUSTER, del paquete SAS®.

Cuadro 4. Mortalidad de hembras de Rhynchophorus palmarum L., provocada por M. anisopliae inoculado a machos, con base en diferentes métodos de aplicación y diferente relación macho/hembra. Villavicencio, Meta, Colombia. 2017.

Table 4. Female mortality of Rhynchophorus palmarum L. caused by M. anisopliae inoculated to males based on different application methods and different male/female ratio. Villavicencio, Meta, Colombia. 2017.

\begin{tabular}{ccccc}
\hline Tratamiento & Cepa & Método de aplicación & Relación macho:hembra & Mortalidad (\%)* \\
\hline 10 & CPMa1104 & Polvo & $1: 1$ & 100,00 \\
11 & CPMa1104 & Polvo & $1: 5$ & 100,00 \\
12 & CPMa1104 & Polvo & $1: 10$ & 100,00 \\
7 & Mt008 & Polvo & $1: 1$ & 90,00 \\
8 & Mt008 & Polvo & $1: 5$ & 63,33 \\
9 & Mt008 & Polvo & $1: 10$ & 63,33 \\
1 & Mt008 & Inmersión & $1: 1$ & 30,00 \\
4 & CPMa1104 & Inmersión & $1: 1$ & 30,00 \\
3 & Mt008 & Inmersión & $1: 10$ & 23,33 \\
5 & CPMa1104 & Inmersión & $1: 5$ & 20,00 \\
6 & CPMa1104 & Inmersión & $1: 10$ & 20,00 \\
2 & Mt008 & Inmersión & $1: 5$ & 16,67 \\
13 & Control & & $1: 1$ & 13,33 \\
15 & Control & & $1: 10$ & 13,33 \\
14 & Control & & $1: 5$ & 10,00 \\
\hline
\end{tabular}

*Medias ajustadas por mínimos cuadrados / *Least-square adjusted averages.

En cuanto a la relación del número de machos confinados con diferente número de hembras, se observó mayor mortalidad de hembras cuando la relación fue de 1:1, lo cual sugiere que hay una mejor diseminación de las esporas del hongo, cuando el contacto se da en esta proporción.

Para la mortalidad de hembras de R. palmarum, se conformaron tres grupos: el grupo 3 constituido por los tratamientos 7, 10, 11 y 12, fue el que mostró mayor mortalidad de hembras, que correspondieron a las cepas CPMa1104 inoculada a los machos en polvo, en las tres relaciones macho/hembra evaluadas, y la Mt008 aplicada a los machos en polvo, en la relación macho/hembra 1:1; el grupo 2 reunió los tratamientos 8 y 9 de mortalidad 
intermedia, es decir, la cepa Mt008 inoculada a los machos en polvo en las relaciones macho/hembra 1:5 y 1:10; y el grupo 1 congregó los tratamientos con menor mortalidad que corresponden a las cepas Mt008 y CPMa1104, aplicadas a los machos mediante el procedimiento de inmersión y al tratamiento control absoluto sin inoculación del entomopatógeno (Cuadro 5).

Cuadro 5. Estadísticas descriptivas por grupos para la mortalidad de hembras de Rhynchophorus palmarum L. Villavicencio, Meta, Colombia. 2017.

Table 5. Descriptive statistics by groups for female mortality of Rhynchophorus palmarum L. Villavicencio, Meta, Colombia. 2017.

\begin{tabular}{|c|c|c|c|c|c|c|c|}
\hline Grupo & Cepa & $\begin{array}{l}\text { Método } \\
\text { aplicación }\end{array}$ & $\begin{array}{c}\text { Relación } \\
\text { macho:hembra }\end{array}$ & Media & Mín & Máx & C.V. (\%) \\
\hline \multirow{9}{*}{1} & Mt008 & Inmersión & $1: 1$ & 19,63 & 10,00 & 30,00 & 20,13 \\
\hline & Mt008 & Inmersión & $1: 5$ & & & & \\
\hline & Mt008 & Inmersión & $1: 10$ & & & & \\
\hline & CPMa1104 & Inmersión & $1: 1$ & & & & \\
\hline & CPMa1104 & Inmersión & $1: 5$ & & & & \\
\hline & CPMa1104 & Inmersión & $1: 10$ & & & & \\
\hline & Control & & $1: 1$ & & & & \\
\hline & Control & & $1: 10$ & & & & \\
\hline & Control & & $1: 5$ & & & & \\
\hline \multirow{2}{*}{2} & Mt008 & Polvo & $1: 5$ & 63,33 & 63,33 & 63,33 & 0,00 \\
\hline & $\mathrm{Mt} 008$ & Polvo & $1: 10$ & & & & \\
\hline \multirow{4}{*}{3} & Mt008 & Polvo & $1: 1$ & 97,50 & 90,00 & 100,00 & 5,13 \\
\hline & CPMa1104 & Polvo & $1: 1$ & & & & \\
\hline & CPMa1104 & Polvo & $1: 5$ & & & & \\
\hline & CPMa1104 & Polvo & $1: 10$ & & & & \\
\hline
\end{tabular}

C.V.: coeficiente de variación / C.V.: variation coefficient.

\section{Discusión}

En cuanto a la determinación de la patogenicidad de $M$. anisopliae sobre adultos de $R$. palmarum, la cepa CPMa1104 resultó ser la más patogénica, al causar mortalidades del 80 y $90 \%$, seguida de la cepa Mt008 con mortalidad de 73,3\%, lo cual confirma la alta eficacia de M. anisopliae sobre el control de adultos de R. palmarum reportada en trabajos previos (Mora, 2007; Aldana et al., 2011; Alvarado, et al., 2013). Las cepas Mt040 y Bv063, presentaron valores medios de control; la cepa CPMa1105 y el producto comercial evaluado, presentaron bajos porcentajes de mortalidad; este resultado se explica por la especificidad de los aislamientos seleccionados y por la patogenicidad de cada uno de ellos para el control de adultos de R. palmarum; según Valencia et al. (2011) esta variación en los resultados entre cepas puede estar relacionada con la capacidad de adherencia y penetración de los conidios, así como por su actividad patogénica y la capacidad inmunológica del insecto.

En Colombia, en Cenipalma se reportó hasta un $87 \%$ de control (Aldana et al., 2011), y en Costa Rica se obtuvieron mortalidades de adultos de $R$. palmarum hasta del $100 \%$, cuando se aplicaron diferentes cepas de $M$. 
anisopliae (Esteves y Cedeño, 2008). Mortalidades del $100 \%$ se obtuvieron después de treinta días sobre adultos de $R$. ferrugineus con concentraciones de $1 \times 10^{8}$ conidias $\mathrm{ml}^{-1}$ del hongo M. anisopliae (Gindin et al., 2006).

La formulación en polvo también ha sido comprobada como forma más eficiente de diseminación por varios autores; por ejemplo, Gindin et al. (2006) encontraron resultados similares a los del presente trabajo sobre el efecto del método de inoculación de $M$. anisopliae y $B$. bassiana, al evaluar varias cepas de entomopatógenos sobre Rhynchophorus ferrugineus, y afirmaron que la formulación a base de arroz en polvo seco aceleró la mortalidad adulta al $100 \%$ en dos a tres semanas, mientras que en la suspensión de esporas en agua la mortalidad se logró hasta las cuatro a cinco semanas.

En cuanto a la diseminación del entomopatógeno de machos a hembras de $R$. palmarum se han obtenido similares resultados en esta investigación, donde la formulación en polvo ha producido mortalidades en las hembras significativamente mayores a los tratamientos de inmersión y no se han encontrado diferencias significativas con relación a la proporción de machos y hembras confinados por tratamiento (Gindin et al., 2006); esto se explica porque la formulación en polvo permite movilidad y mejor contacto de los individuos con las esporas del entomopatógeno, ello favorece la adherencia de mayor cantidad de esporas en el integumento del insecto comparado con la suspensión líquida, tal como afirmaron Arrubla et al. (2008), quienes encontraron mayor adherencia de esporas en el cuerpo de brocas, con M. anisopliae en polvo, cuando los adultos de Hypothenemus hampei caminaron sobre la superficie tratada con esporas del hongo.

La dispersión de entomopatógenos mediante los mismos individuos infectados se considera una importante estrategia de autodiseminación (Arrubla et al., 2008; Ferreira et al., 2014; Suárez-Núñez et al., 2014). En relación con la autodiseminación del entomopatógeno por machos de $R$. palmarum inoculados hacia hembras sin inocular, Ferreira (2002) observó que los machos inoculados con una suspensión de $10^{9}$ conidias $\mathrm{ml}^{-1}$ del hongo B. bassiana, liberados en campo quincenalmente durante tres años, redujeron la población de la plaga en $58 \%, 38 \%$ y $69 \%$ respectivamente, en cada año. Los resultados obtenidos en el presente trabajo con mortalidades de hembras de $R$. palmarum hasta del $100 \%$, comprueban la auto diseminación de esporas de machos hacia hembras, lo cual podría ser una solución viable y aplicable en programas de manejo integrado de la plaga, con métodos similares a los evaluados por Ferreira (2002). Posteriormente, Ferreira et al. (2014) obtuvieron resultados en los cuales los machos de $R$. palmarum transmitieron hasta en $95 \%$ los conidios del hongo B. bassiana hacia las hembras, con base en el instinto de agregación de la especie, además, evaluaron tratamientos en que los machos fueron inoculados y después de 24 h liberados para que diseminaran las esporas del hongo. Una reducción hasta del 72,2 \% en la población de la plaga, la obtuvieron Santos et al. (2014), con liberaciones quincenales en campo de machos inoculados con $M$. anisopliae en polvo, colocado en trampas de diseminación diseñadas para tal fin, con atrayente de caña de azúcar; dichos resultados se consideran promisorios como una estrategia de diseminación de hongos entomopatógenos, con los machos de la plaga, para el control de las poblaciones en campo.

\section{Conclusiones}

Al evaluar la eficacia de varias cepas promisorias de M. anisopliae y B. bassiana a diferentes concentraciones, se verificó que estos entomopatógenos afectan adultos del picudo $R$. palmarum y la mayor patogenicidad se logró con cepas de M. anisopliae.

En la fase 1, la cepa de M. anisopliae CPMa1104, resultó ser la más patogénica, seguida de la cepa Mt008, por tanto, se concluye que estas dos cepas son las más promisorias entre las evaluadas en este trabajo.

En la fase 2 se determinó que machos de R. palmarum inoculados con esporas del hongo M. anisopliae, de las cepas CPMa1104 y Mt008, lograron autodiseminar el entomopatógeno por contacto hacia hembras de la plaga no inoculadas, en condiciones de confinamiento y en diferentes proporciones numéricas de relación macho/hembra. 
La autodiseminación de esporas de $M$. anisopliae por machos de $R$. palmarum inoculados con las cepas CPMa1104 y Mt008 hacia hembras no inoculadas, registró mortalidades de hembras hasta del $100 \%$, en tratamientos de inoculación en polvo, mientras que para los tratamientos de aplicación por inmersión, la mortalidad de hembras fue mucho menor, lo cual permite concluir que bajo las condiciones en que se realizó esta investigación la aplicación en polvo es más eficaz y la diseminación de esporas de machos a hembras de R.palmarum por medio de este método es mayor.

\section{Agradecimientos}

A la Corporación Centro de Investigación en Palma de Aceite (Cenipalma), su coordinador del Programa de Plagas y Enfermedades, Dr. Alex Bustillo Pardey y a la auxiliar de Investigación Angie Marcela Barragán F., por compartir los aislamientos de las cepas CPMa1105 y CPMa1104, de la Colección de Microorganismos Asociados a la Palma de Aceite-Cenipalma (MPA-Cenipalma), con los cuales se adelantó la presente investigación.

A la Corporación Colombiana de Investigación Agropecuaria AGROSAVIA por facilitar sus instalaciones, laboratorios y sus cultivos comerciales de palma aceitera en el Centro de Investigación La Libertad, para la ejecución de los experimentos, y al Dr. Bernhard L. Lohr por su apoyo incondicional y suministro de insectos e información para el desarrollo de los experimentos.

\section{Literatura citada}

Agronet (Red de Información y Comunicación del Sector Agropecuario de Colombia). 2014. Sistema de estadísticas agropecuarias. Agronet, COL. http://www.agronet.gov.co/Documents/Coco.pdf (consultado 27 abr. 2017).

Aldana, R.C., J.A. Aldana, y O.M. Moya, 2007. Manejo del picudo Rhynchophorus palmarum L. (Coleoptera: Curculionidae). Instituto Colombiano Agropecuario, Bogotá, COL.

Aldana, R.C., J.A. Aldana, y O.M. Moya. 2010. Biología, hábitos y manejo de Rhynchophorus palmarum. Boletín técnico No. 23. Cenipalma, y Fedepalma, Bogotá, COL.

Aldana, R.C., J.A. Aldana, y O.M. Moya. 2011. Manejo del picudo Rhynchophorus palmarum L. Coleoptera: Curculionidae. Cenipalma, Bogotá, COL.

Alvarado, H.L., L.G. Montes, H. Gomes, A.E. Bustillo, y E. Mesa. 2013. Patogenicidad de cepas de Metarhizium anisopliae (L.) y Beauveria bassiana sobre Rhynchophorus palmarum. Palmas 34(2):15-24.

Arrubla M., R. Cárdenas, y F. Posada. 2008. Adherencia de las esporas de Beauveria bassiana formuladas en polvo y líquido sobre la broca del café. Rev. U.D.C.A Actual. Divulg. Cient.11(1):123-133.

Bustillo, A.E., M.G. Bernal, P. Benavides, and B. Chaves. 1999. Dynamics of Beauveria bassiana and Metarhizium anisopliae infecting Hypothenemus hampei (Coleoptera: Scolytidae) populations emerging from fallen coffee berries. FL Entomol. 82:491-498.

Calvache, H., A. Mejía, M. Hernández, y J. Muñoz. 1994. Acción de Metamasius hemipterus L. (Coleoptera: Curculionidae) en la transmisión del anillo rojo de la palma aceitera. Palmas 15(4):17-22.

Castrillón, C. 2000. Distribución de las especies de picudo del plátano y evaluación de entomopatógenos nativos en el departamento de Risaralda. CORPOICA, Manizales, COL.

Chinchilla, C.M. 1988. El síndrome del anillo rojo-hoja pequeña en palma aceitera y cocotero. OPB-CB 2(4):113-136. 
Costa, F., J.A. Sacramento, L. Melo, V. Rossi, N. Le-Coustour, M. Lummerzheim, J.I. Lacerda, and R. Motta. 2011. Mass trapping and biological control of Rhynchophorus palmarum L.: A hypothesis based on morphological evidences. EntomoBrasilis 4(2):49-55. doi:10.12741/ebrasilis.v4i2.85

Dangon, L. 2014. Palma de aceite: compromiso con el progreso y la convivencia. Presentado en: XLII Congreso Nacional de Cultivadores de Palma de Aceite. 3 jun. Cali, COL.

Esteves, E.F., y R.J. Cedeño. 2008. Susceptibilidad de Rhynchophorus palmarum (L.) (Coleoptera: Curculionidae) a cepas de hongos entomopatógenos. Tesis pregrado. Universidad EARTH, Guácimo, CRI.

Faleiro, J.R. 2006. A review of the issues and management of the red palm weevil Rhynchophorus ferrugineus (Coleoptera: Rhynchophoridae) in coconut and date palm during the last one hundred years. Int. J. Trop. Insect Sci. 26(3):135-154. doi:10.1079/IJT2006113

FAO. 2013. Oportunidad para el coco de Centroamérica. Pronostican en Asia una crisis de producción de coco que podría abrir mercados para los productores centroamericanos. CentroamericaData, MEX. https:/www.centralamericadata.com/es/ article/home/Oportunidad_para_el_coco_de_Centroamrica (consultado 27 nov. 2018).

FAOSTAT. 2015. Cultivos. FAO, Roma, ITA. http://www.fao.org/faostat/es/\#data/QC (consultado 10 abr. 2019).

FEDEPALMA (Federación Nacional de Cultivadores de Palma de Aceite). 2016. Desempeño del sector palmero colombiano. Julio de 2016. FEDEPALMA, COL. http://web.fedepalma.org/sites/default/files/files/18072016_Desempen\%CC\%83o_ sector_2015_2016.pdf (consultado 27 abr. 2017).

Ferreira, J.M. 2002. Controle biológico do agente transmissor do nemátoide causador do anel-vermelho-do-coqueiro. Circular técnica 31. Embrapa, BRA. www.cpatc.embrapa.br/download/CT31.pdf (consultado jul. 2018).

Ferreira, J.M., A.V. Teodoro, A. Negrisoli, e E.C. Guzzo. 2014. Manejo integrado da broca-do-olho-do-coqueiro Rhynchophorus palmarum L. (Coleoptera: Curculionidae). Comunicado Técnico 141. Docplayer, BRA. https://docplayer.com.br/11171112Comunicado141-tecnico.html (consultado feb. 2019).

Genty, P. 1988. Manejo y control de plagas en palma africana. En: B. Ramakrishna, editor, VI Seminario sobre problemas fitopatológicos de la palma africana. IICA, BID, y PROCIANDINO, COL. p. 101-112.

Gindin, G., S. Levski, I. Glazer, and V. Soroker. 2006. Evaluation of the entomopathogenic fungi Metarhizium anisopliae (L.) and Beauveria bassiana against the red palm weevil Rhynchophorus ferrugineus. Phytoparasitica 34:370-379. doi.10.1007/BF02981024

Griffith, R. 1987. Red ring disease of coconut palm. Plant Dis. 71:193-196. doi:10.1094/PD-71-193

Godonou, I., K.R. Green, K.A. Oduro, C.J. Lomer, and K. Afreh-Nuamah. 2000: Field evaluation of selected formulation of Beuveria bassiana for the management of the banana weevil (Cosmopolites sordidus) on plantain (Musa spp.). Biocontrol Sci. Technol. 10:779-788. doi.10.1080/09583150020011726

González-Cárdenas, A. 2016. La agroindustria de la palma de aceite en América. Palmas 37(Esp. II):215-228.

González, M.T., F.F. Posada, y A.E. Bustillo. 1993. Bioensayo para evaluar la patogenicidad de Beauveria bassiana (Bals.) Vuill. Sobre la Broca del Café, Hypothenemus hampei (Ferrari). Rev. Colom. Entomol. 19(4):123-130.

Gopal, M., A. Gupta, B. Sathiamma, and C.P. Nair. 2002. Microbial pathogens of the coconut pest Oryctes rhinoceros: influence of weather factors on their infectivity and study of their coincidental ecology in Kerala, India. World J. Microbiol. Biotechnol. 18:417-421. doi:10.1023/A:10155406

Grueso, W., y C. Betancourth. 2009. Evaluación de erradicación del cocotero para el manejo del anillo rojo Bursaphelenchus cocophilus - Gualpa Rhyncophorus palmarum (Coleoptera: Curculionidae) en Tumaco, Nariño. Rev. Cienc. Agríc. 26:171-185 
Hagley, A.C. 1963. The role of the palm weevil, Rhynchophorus palmarum, as a vector of red ring disease of coconuts. I. Results of preliminary investigations. J. Econ. Entomol. 56:375-380. doi:10.1093/jee/56.3.375

Kreutz, J., G. Zimmermann, and O. Vaupel. 2004. Horizontal transmission of the entomopathogenic fungus Beauveria bassiana among the spruce bark beetle, Ips typographus (Col., Scolytidae) in the laboratory and under field conditions. Biocontrol Sci. Technol. 14:837-848. doi.10.1080/788222844

MADR (Ministerio de Agricultura y Desarrollo Rural), ICA (Instituto Colombiano Agropecuario), y FEDEPALMA (Federación Nacional de Cultivadores de Palma de Aceite). 2014. Ministerio de Agricultura, ICA y FEDEPALMA, lanzan campaña contra la pudrición del cogollo. MADR, COL. https://www.minagricultura.gov.co/noticias/Paginas/Ministerio-de-Agricultura,ICA-y-FEDEPALMA,-lanzan-campa\%C3\%B1a-contra-la-Pudrici\%C3\%B3n-del-cogollo.aspx (consultado 27 abr. 2017 ).

Mora, C.A. 2007. Efecto de la aplicación de Metarhizium anisopliae (Hyphomycetes) y peremetrina sobre la densidad de adultos de Rhynchophorus palmarum (Coleoptera: Curculionidae) y palmas atacadas por Bursaphelenchus cocophilus (Tylenchidae) en campo. Tesis Lic., Universidad de Colima, MEX.

Mora, L.S., H.H. Calvache, y M. Avila. 1994. Diseminación de Radinaphelenchus cocophilus, agente causal del anillo rojo de la palma aceitera en San Carlos de Guaroa. Palmas 15(1):15-27.

Nájera-Rincón, M.B., M. García-Martínez, R.L.. Crocker, V. Hernández-Velázquez, y L.A. Rodríguez. 2005. Virulencia de Beauveria bassiana y Metarhizium anisopfiae, nativos del Occidente de Mexico, contra larvas de Phyllophaga crinita (Coleoptera: Melolonthidae) bajo condiciones de laboratorio. Fitosanidad 9:33-36.

Oehlschlager, C. 2007. Optimizing trapping of palm weevils and beetles. Acta Hort. 736:347-368. doi:10.17660/ ActaHortic.2007.736.33

Pedrini, N., M.L. Villaverde, C.B. Fuse, G.M. Dal-Bello, and M.P. Juárez. 2010. Beauveria bassiana infection alters colony development and defensive secretions of the beetles Tribolium castaneum and Ulomoides dermestoides (Coleoptera: Tenebrionidae). J. Econ. Entomol. 103:1094-1099. doi:10.1603/EC10072

Picado, H.C. 2017 Palma aceitera como política de estado en Centroamérica. Grain. https://www.grain.org/es/article/ entries/5663-palma-aceitera-como-politica-de-estado-en-centroamerica (consultado 25 oct. 2018).

Prior, C., P. Jollands, and M. Arura. 1985. The infectivity of Metarhizium anisopliae to two insects pests of coconuts. J. Invertebr. Pathol. 45:187-194. doi:10.1016/0022-2011(85)90008-4

Prior, C., P. Jollands, and P. Le. 1988. Infectivity of oil and water formulations of Beauveria bassiana (Deuteromycotina: Hyphomycetes) to the coco weevil pest Pantorhytes plutus (Coleoptera: Curculionidae). J. Invertebr. Pathol. 52:66-72. doi:10.1016/0022-2011(88)90103-6

SAS Institute Inc. 2016. User's guide version 9.4. SAS Institute Inc., Cary, NC, USA.

Santos, J., V.A. Teodoro, A. Negrisoli, e E. Guzzo. 2014. Manejo integrado da broca-doolho-do-coqueiro Rhynchophorus palmarum L. (Coleoptera: Curculionidae). Comunicado Técnico 141. Embrapa, Aracaju, BRA.

Suárez-Núñez, J., y H. Cortez-Madrigal. 2014. Transmisión horizontal de Beauveria bassiana en poblaciones controladas de Bactericera cockerelli (Hemiptera: psyllidae). Entomol. Mex. 1:338-343.

Tinzaara, W., C.S. Gold, M. Dicke, A. Van-Huis, C.M. Nankinga, G.H. Kagezi, and P.E. Ragama. 2007. The use of aggregation pheromone to enhance dissemination of Beauveria bassiana for the control of the banana weevil in Uganda. Biocontrol Sci. Technol. 17:111-124. doi:10.1080/09583150600937089

Valencia, C., S. Pérez, R. Aldana, E. Mesa, y H. Oliveira, 2011. Patogenicidad de hongos entomopatógenos del género Metarhizium sobre larvas de Strategus aloeus L. (Coleoptera: Scarabaeidae), en condiciones de laboratorio. Palmas. 32(4):30-40. 